\title{
Bat algorithm (BA): review, applications and modifications
}

\author{
Amar Yahya Zebari' ${ }^{1}$, Saman M. Almufti²*, Chyavan Mohammed Abdulrahman ${ }^{3}$ \\ ${ }^{I}$ Department of Statistics, College of Science, Van Yuzuncu Yil University, Van, Turke \\ ${ }^{2}$ Department of Computer Science and Information Technology, College of Computer Science \& Information Technology, \\ Nawroz Uni-versity, Duhok, Iraq \\ ${ }^{3}$ College of Physical Education and Sport Sciences, Duhok University, Duhok, Iraq \\ *Corresponding author E-mail:Saman.Almofty@gmail.com
}

\begin{abstract}
Generally, Metaheuristic algorithms such as ant colony optimization, Elephant herding algorithm, particle swarm optimization, bat algorithms becomes a powerful methods for solving optimization problems. This paper provides a timely review of the bat algorithm and its new variants.

Bat algorithm (BA) is a Swarm based metaheuristic algorithm developed in 2010 by Xin-She Yang, BA has been inspired by the foraging behavior of micro bats, algorithm carries out the search process using artificial bats as search agents mimicking the natural pulse loudness and emission rate of real bats. It has become a powerful swarm intelligence method for solving optimization prob-lems over continuous and discrete spaces. Nowadays, it has been successfully applied to solve problems in almost all areas of opti-mization, and it found to be very efficient. As a result, the literature has expanded significantly, a wide range of diverse applications and case studies has been made base on the bat algorithm.
\end{abstract}

Keywords: Swarm Intelligence (SI); Bat Algorithm (BA); Literature Review; Metaheuristic Algorithm.

\section{Introduction}

The increasing complexity of real world problems causes the researchers to search for efficient techniques. Divide and conquer techniques are efficient way to solve large and complex problems which has been a practice in research since long time such as (NP-hard problems).

Modern optimization algorithms are often nature-inspired, typically based on swarm intelligence. The ways for inspiration are diverse and consequently algorithms can be many deferent types. However, all these algorithms tend to use some specific characteristics for formulating the key updating formulae [1].

Swarms such as bee colonies, ant colonies, mosquito swarms, fish schools, Bat swarm, have relatively simple behaviors individually, but with amazing capability of co-operations and organizing their actions, they represent a complex and highly structured social organization. Swarm Intelligence (SI) is the field of studying and designing well-organized computational intelligent interactive multi-agent systems that cooperate togather to achieve a specific goal and to solving complex optimizations problems by using the behavior of real living swarms such as birds, fish, bats, and ants [1] [2], SI is a part of Artificial Intelligence (AI) introduced by Jing Wang and Gerardo Beni in 1989 in the global optimization framework as a collection of algorithms for controlling robotic swarms [1 - 3].

Bat algorithm (BA) was introduced by Yang in 2010 [1][6] [7], It simulates the echolocation behavior of microbats as microbats can generate high echolocation. The Bat produces a very high sound to detect its prey which echoes back with some frequency. Echolocation is a process of detecting an object by reflected sound. It is used to know how far the prey is from background object. By observing the bounced frequency of sound, bats are able to distinguish between the prey and obstacle and can sense the distance between them in their nearby surroundings. They fly randomly with some velocity, frequency and sound (loudness) to search for food. Solution of objective function is to find prey at minimum distance. The frequency and zooming parameters maintain the balance between exploration and exploitation processes. The algorithm continued till convergence criteria are satisfied[1] [4].

\section{Swarm intelligence algorithms}

Swarm intelligence(SI), which is an artificial intelligence(AI)field,isconcernedwiththedesigningofintelligent interactivemultiagentsystemsthatcooperatetogather toachieveaspecificgoal.Swarmintelligenceisdefined byDorigo M as"The emergent collective intelligence of groupsofsimpleagents" [1] [5]. Swarm-based algorithmsareinspiredfrombehaviorsofsomesocial livingbeings(insects,animal,andbacteria's)inthe nature,suchasants,birds,bats,bees,termites, and fishes.Themostremarkablefeaturesofswarmsystems areSelf-organizationanddecentralizedcontrolthat naturallyleadstoanemergentbehaviorinthecolony. Emergentbehaviorisaninteractivepropertythat emergesalocalinteractionamongallsystem components (agents) and it is not 
possible to be achieved alonebyany agentinthesystem [1] [3].In computersciencetherearemanyalgorithmsthatare designedasaninspirationofrealcollectivebehavior systemsinthenature,swarmintelligencealgorithms includesAntColonyOptimization(ACO),Particle Swarm Optimization (PSO), Artificial Bee Colony (ABC), ArtificialImmuneSystem,Batalgorithm,Bacterial Foraging, Stochastic diffusion search, Glowworm Swarm Optimization,Gravitationalsearchalgorithm,Cat Swarm Optimization, and other optimization algorithms [1] [3]. Swarmintelligenceworksontwobasicprinciples:self- organization and stigmergy (e.g., Fig. 1).

1) Self-organization: This can be characterized by three parameters like structure, multi stability and state transitions. In swarms, interpreted the self-organization through four characteristics: (i) positive feedback,(ii) negative feedback, (iii) fluctuations, and (iv) multiple interactions.

2) Stigmergy: It means stimulation by work. Stigmergy is based on three principles:(i) work as a behavioral response to the environmental state, (ii) an environment that serves as a work state memory(iii)work that does not depend on specific agents.

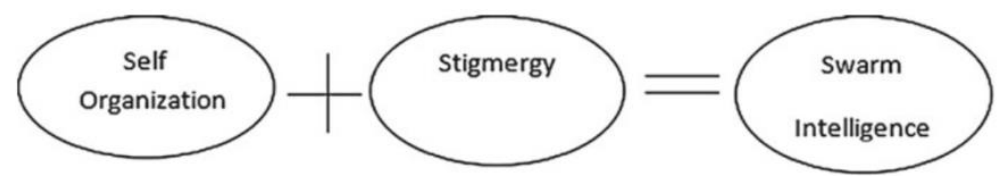

Fig. 1: Swarm Intelligence Basic Principles.

AntColonyOptimization(ACO)isapopulationbased optimizationalgorithmdevelopedby MarcoDorigo as aninspirationofthebehaviorofantsinfindingthe optimalway(bestpath)betweentheirnestandafood source [3].TheBatalgorithmis anatural inspiredmetaheuristicalgorithmfor globaloptimization problems, it was inspired by theecholocationbehaviorofmicrobats, withvarying pulseratesofemissionandloudness [6]. The Bat algorithm (BA) was developedby thescientist [6].Artificial beecolonyalgorithm(ABC)isanaturalinspired metaheuristicoptimizationalgorithmbasedonthe intelligentforagingbehaviorofhoneybeeswarm, proposedbythe scientistKarabogaforsolving combinatorialoptimization problems(MarcoDorigo, Thomas Stu, 2004). Particle Swarm Optimization (PSO) is a population based optimization algorithm developed by EberhartandKennedyasaninspiredbybirdflocks' behavior when searching for food [2].Thetravelsalesmanproblem(TSP)whichisan NP-hardproblemthatisimpossibletofindthe optimal tour with in an optimal time has been studied extensively overthepastseveraldecades.InthispaperACOand PSOareusedtofindthesolutionofTSP[7][8],solutioninarelativelylesstime(shortexecutiontime) insteadoffindinganoptimalsolutionwhichisnoteasy to compute and have a long execution time [9][10]. UsingexactalgorithmsforNP-hardproblemsare not preferable, because they takes unbounded (long) time toexecute,forthisreasonresearchesoftenuse approximatesmethods, whichtriestoobtainanear $\quad$ optimal solutionforNPhardproblemsinasignificantly short bounded time [1].

\section{Bat algorithm (BA)}

Bats are eye-catching animals and their higher potential of echolocation has engrossed interest of scholars from various arenas. Echolocation mechanism is a kind of sonar: bats, mainly micro-bats, create a loud and short pulse of sound and figure out the distance of an object by using the echo reruns back to their ears [5]. This remarkable positioning method makes bats being able to decide the difference between an obstacle and a prey, allowing them to hunt even in whole darkness [1], [5].

Motivated by the conduct of the batsXin-She Yang has developed the Bat Algorithm.

Batalgorithm (BA)isapopulationbasedmetaheuristic algorithmproposedbyYangin2010forsolving continuous optimization problems [1] [12]. The basicBAalgorithmisbio-inspiredonthe bio-sonarorecholocationcharacteristicsofbats.In nature,batsreleaseultrasonicwavestotheenvironment around it for the purposes of hunting or navigation. After theemission of these waves,it receives theechoesofthe waves,andbasedonthereceivedecho theylocate themselvesandidentifyobstaclesintheirwaysand preys as shown in figure 2.Furthermore,eachagentinswarmiscapableof finding the most "nutritious" areas or moving towards a previousbestlocationfoundbytheswarm [11].Batalgorithmhasshowedgrate efficiencyinfinding solutionincontinuousoptimization problems [6].

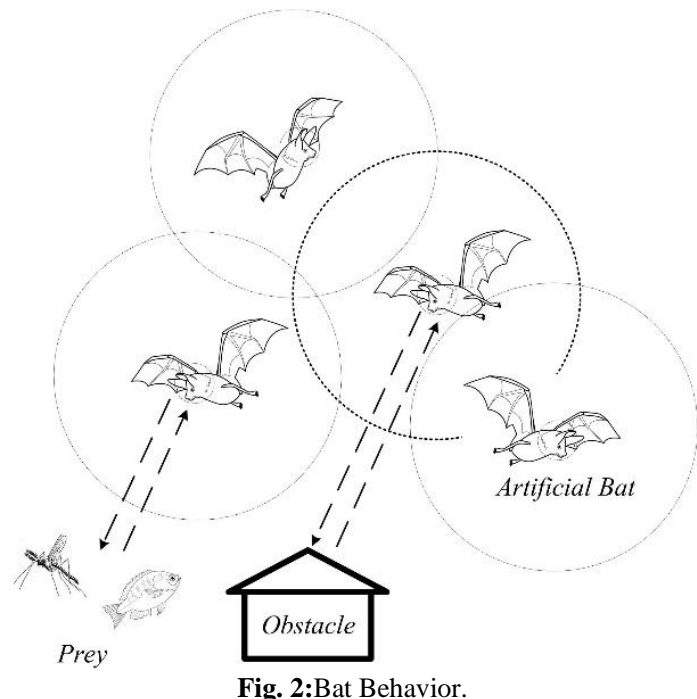

\subsection{Bat flowchart and pseudocode}

First initialize the bat population then we have to define the pulse frequency, after that we initialize pulse rates and loudness in which we define maximum no of iterations, if result is better than new values will generate and values will updated in velocities. In this random 
values will generate if solution is yes then we have to select the best solution ,if not then program will move back .when new solutions form in random values it accepts it and find the best current value and output is form[5]. Figure 3 shows the Flowchart of BA.

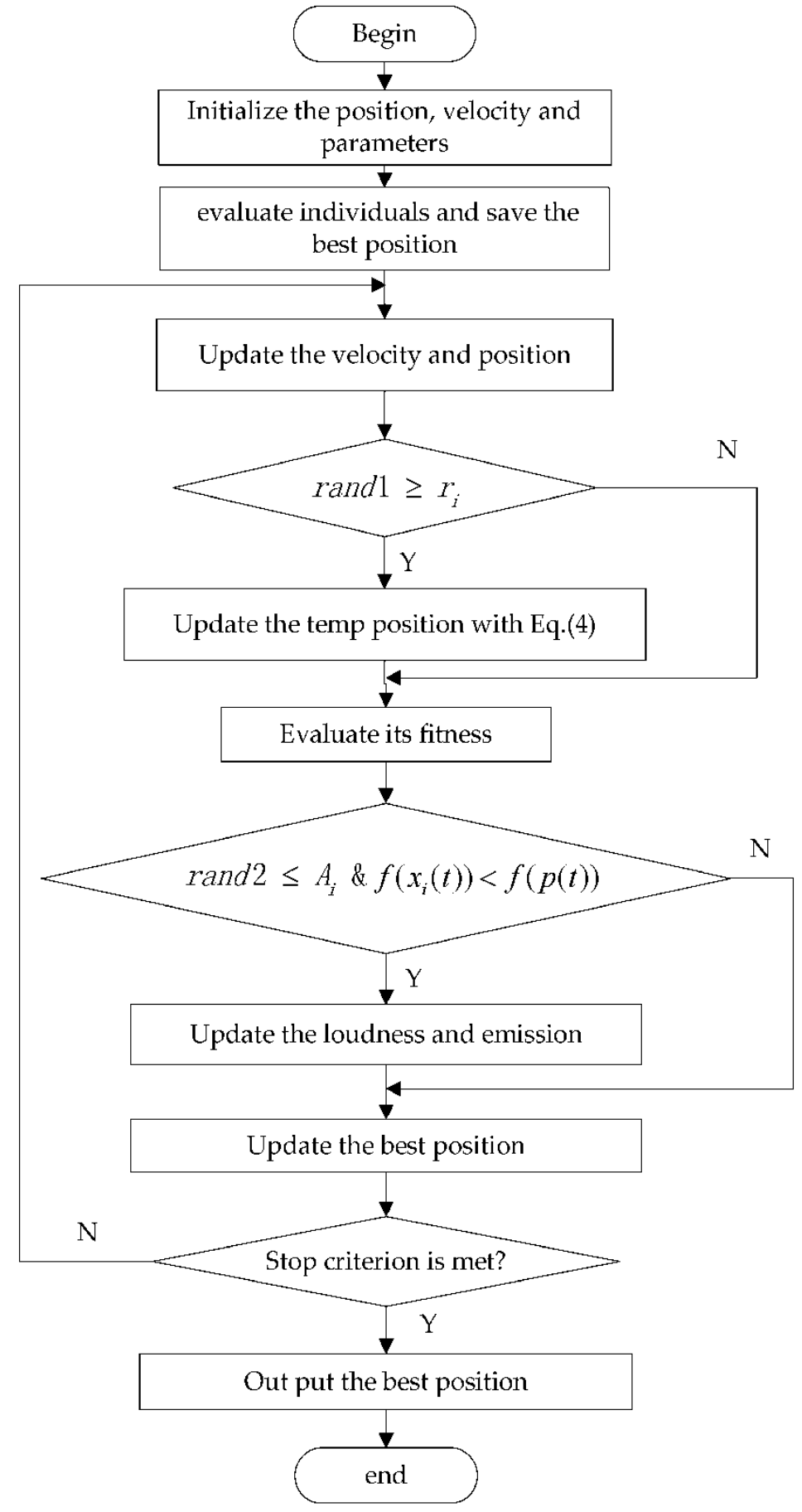

Fig. 3: BA Flowchart.

BApseudo code is shown below in (Algorithm 1):

Algorithm 1: Pseudocode of the basic BA:

Define the objective function $\mathrm{f}(\mathrm{x})$,

Initialize the bat population $X=x 1, x 2, \ldots, x n$,

for each bat $\mathrm{xi}$ in the population doInitialize the pulse rate ri, velocity vi and loudness $\mathrm{Ai}$,

Define the pulse frequency fi at xi,

end

repeat

for each bat xi in the population do

Generate new solutions through Equations 1, 2 and 3,

if rand $>$ ri then

Select one solution among the best ones,

Generate a local solution around the best one, end

if rand $<\mathrm{Ai}$ and $\mathrm{f}(\mathrm{xi})<\mathrm{f}\left(\mathrm{x} \_\right)$then 
Accept the new solution, Increase ri and reduce Ai,

end

end

until termination criterion not reached,

Rankthebatsandreturnthecurrentbestbatofthe population,

Equations for Generating new solutions

First of all, the starting position, velocity, and frequency are initialized for every bat . For each time step t, being $\mathrm{T}$ the limit of iterations, the movement of the virtual bats is specified by updating their velocity and position by means of equations 1, 2, and 3 as follows [1].

$$
\begin{gathered}
f_{i}=f_{\min }+\left(f_{\min }-f_{\max }\right) \beta_{(1)} \\
v_{i}^{t}=v_{i}^{t-1}+\left[x_{i}^{t-1}-x_{*}\right] f_{i}(2) \\
x_{i}^{t}=x_{i}^{t-1}+v_{i(3)}^{t}
\end{gathered}
$$

Where $\beta \in[0,1]$ is a random vector drawn from a uniform distribution, $f_{\mathrm{i}}$ denotes frequency of each bat, here, $x_{s}$ is the current global best solution (location) which is located after comparing all the solutions among all $\mathrm{n}$ bats, at each iteration. After the position updating of bats, a random number is generated, if the random number is larger than the pulse emission rate $x_{\mathbb{1}}^{x}$,a new position will be generated around the current best solutions, and it can be represented by equation (4) [1].

$$
x_{\text {new }}=x_{\text {old }}+\varepsilon A^{t}(4)
$$

Where, $\in[-1,1]$, is a random number, while $A^{\mathrm{t}}$ is the average loudness of all the bats at current iteration. Furthermore, the loudness $A^{\mathrm{t}}$ and the pulse emission rate $r_{\mathrm{i}}^{\mathrm{n}}$ will be updated and a solution will be accepted if a random number is less than loudness $A^{\mathrm{i}}$ and $f\left(x_{i}\right)<f\left(x_{*}\right) \cdot A^{i}$ and $x_{i}^{x}$ are updated by (5).

$$
A_{i}^{t+1}=\alpha A_{i}^{t}, \quad r_{i}^{t+1}=r_{i}^{0}[1-\exp (-\gamma t)]
$$

Where a and y are constants, the algorithm iterates until the termination criteria is met.

\subsection{Application}

\begin{tabular}{|c|c|c|}
\hline Area/Applications & Author & References \\
\hline Travelling salesman problem & Saman & [1] \\
\hline classification, wireless sensor, data mining & Yang et al, & [13] \\
\hline solve multi-objective problems & Yang et al, & [14] \\
\hline brushless direct current (DC) wheel motor problem & Bora et al, & [15] \\
\hline robust turning of power system stabilizer for small signal stability enhancement & Sambariya et al, & [16] \\
\hline load frequency control & Sathya et al, & [17] \\
\hline node localization of wireless sensor networks based & Sun et al, & [18] \\
\hline low energy adaptive clustering hierarchy protocol based & Cao et al, & [19] \\
\hline support vector data description(SVDD)(CBA-SVDD)to design effective descriptions of data & Hamidzadeh et al, & [22] \\
\hline facial feature selection & Alsalibi et al & [23] \\
\hline vector machine (SVM) parameters that reduce the classification error & Yang et al, & [24] \\
\hline biomedical research & Tharwat et al. & [25] \\
\hline Fuel arrangement optimization of reactor core & Kashi et al. & [26] \\
\hline Multilevel image thresholding & Alihodzic and Tuba & [27] \\
\hline Economic dispatch & Latif and Palensky & [28] \\
\hline Feature selection & Taha et al. & [29] \\
\hline Application in Multiple UCAVs & $\mathrm{Li}$ and Peng & [31] \\
\hline Design of a conventional power system stabilizer & Sambariya and Prasad & [32] \\
\hline Toy model of protein folding & Cai et al. & [33] \\
\hline Design of skeletal structures & Kaveh and Zakian & [34] \\
\hline face recognition & Alsalibi & [35] \\
\hline
\end{tabular}

Since the original bat algorithm (BA) has been developed by Yang in2010 [7], bat algorithms and its modifications have been applied in almost every area of optimisation, classifications, image processing, feature selection, scheduling, data mining and others. Table 1will highlight some of the applications of BA [5].

Table 1:BA Applied Erea

\subsection{Modification to bat algorithm (BA)}

To improve the enactment ofBA, many modifications and strategies have been tried, andmany new variants of the bat algorithm are created in the recent literature. Table 2 summarize some the latest variants of bat algorithm. 
Table 2:Bat Algorithm Variants Modifications

\begin{tabular}{llll}
\hline $\begin{array}{l}\text { Modifications } \\
\text { on BA }\end{array}$ & Authors & References & Descriptions \\
\hline $\begin{array}{l}\text { fuzzy bat algo- } \\
\text { rithm }\end{array}$ & Nikov et al, & {$[36]$} & The modified algorithm was experienced on cluster analysis and found to be very effective \\
$\begin{array}{l}\text { binary bat } \\
\text { algorithm }\end{array}$ & $\begin{array}{l}\text { Nakamura et } \\
\text { al, }\end{array}$ & {$[37]$} & $\begin{array}{l}\text { A binary bat algorithm for solving the well known economic load dispatch problem with the valve-point } \\
\text { effect }\end{array}$
\end{tabular}

binary bat algorithm that uses the sigmoid function discrete binary Sabba and variant of BA Chikhi

$\begin{array}{ll}\begin{array}{l}\text { bat algorithm } \\ \text { with mutation }\end{array} & \text { Wang and }\end{array}$

opposition-

based BAT

algorithm

Sabba et al

BAT algorithm with levy flights trajectory

Xie et al

Chaotic BA (CBA)

employed chaos in original

BA

Improving the exploration mechanism of original BA

enhancing the explorative mechanism of BA

bat algorithm with Gaussian walk

Afrabandpey et al.

Gandomi and Yang

Yilmaz et al

Li and Zhou

Cai et al.

cloud model

BA (CBA)

Zhou et al

compact bat

algorithm

(cBA)

self-adaptive

bat algorithm

Fister et al

hybridized the

self adaptive

bat algorithm

Enhanced BA

Fister et al

Yilmaz and

Küçüksill

double sub-

population levy

flight BA

(DLBA)

Doppler Effect

with standard

BA.

Improved BA

(IBA

Jun et al.

Meng et al.

Wang et al
With the help of sigmoid function only binary allowed to new bat's position. They used this variant in feature selection problem

discrete binary variant of BA and tested it on multidimensional knapsack problem. With experimental results they concluded that this discrete binary BA outperformed the standard BA

a new bat algorithm with mutation for image processing. They made two modifications to original BA. First, they used fixed frequency and loudness and second, they added a mutation operator to increase the diversity of the population. They tested it on image processing and found that the proposed algorithm produce good result than standard BA. The standard BA hybridized with differential evolution techniques. This hybridization enhanced the local search capability of original BA.

Sabba et al. improved the convergence speed of BA by embedding the opposition based numbering concept. They tested it against several benchmark functions. Simulation results showed that their approach increases the accuracy and convergence speed of BA.

Xie et al. improved the low accuracy rate and slow convergence speed of BA. They introduced levy flights trajectory which increases the diversity of population, so that the algorithm effectively jump out of local minima. They also used the differential operator to accelerate the convergence speed. The proposed algorithm was tested on typical benchmark functions and they concluded that their approach has superior approximation capabilities in high dimensional space.

Afrabandpey et al. used chaotic sequence for parameter initialization of BA. They called it Chaotic BA (CBA). They studied the effect of different chaotic sequence on convergence behaviour of BA. Simulation result showed that CBA outperforms the BA.

Gandomi and Yang chaos in original BA. They developed four different chaotic BA variants and used 13 different chaotic maps to validate each variant. They concluded that their approach increase the global search mobility of BA.

Yilmaz et al improved the exploration mechanism of original BA. They changed the equation of loudness and pulse emission rate of bats. They tested this modified bat algorithm (MBA) on 15 different benchmark functions and concluded that MBA performs better than BA

$\mathrm{Li}$ and Zhou also enhanced the explorative mechanism of BA by introducing complex value encoding scheme into BA. They update real and imaginary part of complex encoding separately which increases the diversity of population.

They improved the local search capability by introducing the Gaussian walk instead of uniform random walk. They also changed the velocity update equation of BA which results in high population diversity. This approach expands the search dimensions.

They incorporated cloud model concept into BA and called it cloud model BA (CBA). Cloud model has excellent characteristics of representing uncertain knowledge. They remodeled the echolocation model of BA by utilizing the transformation theory of cloud model. They studied that proposed algorithm had good performance on function optimizations

compact bat algorithm (cBA) was developed for limited hardware resources environments. They replaced the design variable of solution search space of BA with a probabilistic representation of the population. Their study showed that this approach can be effectively used in limited memory case.

Fister et al. presented [26] a self-adaptive bat algorithm in which control parameters were self-adapted in the similar way like self-adaptive DE algorithm. They tested it on ten benchmark functions and found that proposed method can be used in continuous optimization efficiently.

Fister et al. hybridized the self adaptive bat algorithm with different DE strategies. These techniques improved the local search capability of the proposed algorithm

local and global search capability of BA was improved by using inertia weight modification, distribution of the population modification, and hybridization with invasive weed optimization algorithm

for enhancing local and global search ability, Jun et al. developed a double sub-population levy flight BA (DLBA) They employed two subgroups namely external subgroup and internal subgroup. Global exploration improved by external subgroup and local exploitation was improved by the internal subgroup. They tested proposed algorithm on several test functions and concluded DLBA can outperform the BA.

Meng et al. introduced the bat's habitat selection and their self-adaptive compensation for Doppler Effect in echoes into the standard BA.

Wang et al improved version of BA called it improved BA (IBA). They combine BA with DE in order to select the best solution in the bat population. They used this algorithm in three dimensional path 
greedy random-

ized adaptive

search proce-

dure with BA

using optimal

forage strategy Cai et al

in BA

quantum be-

haved mean

best position

BA (QMBA)

hybrid multi

objective shuf-

fled BA

(MOsh-BAT)

\section{planning problem and concluded that proposed approach can performed better than BA.}

Zhou et al. successfully integrated the greedy randomized adaptive search procedure and path relinking into the standard BA. They used it in capacitated vehicle routing problem and found it very effective. For solving multi-model numerical problems.

They improved the local search ability by using optimal forage strategy. They also introduced a random disturbance strategy to enhance the global search ability in multi-model environment.

Zhu et al. proposed a quantum behaved mean best position BA (QMBA) for improving the convergence speed of BA. In early stages of this algorithm, the position of each bat updated by current best solution and in later stages, bat's position depends upon the mean best position.

proposed a hybrid multi objective shuffled BA (MOsh-BAT). They combine the features of shuffled

SFLA was combined to form a new optimization algorithm.

\section{Conclusion}

Based on the behavior of Bat a Method has been designed by Yang in 2010 called Bat Algorithm (BA) . This algorithm has proved to be better than other nature inspired algorithm. This algorithm has also been applied to many problems such as: classification and data mining, image process and fuzzy logic etc. BA is a very promising technique which can be further expired for application in many areas. Further, its hybrids can also be developed and tested for various engineering problems.

This paper shows that Batalgorithm(BA)hasbecomeapowerfulnatureinspired metaheuristic algorithm for many continuous and discrete optimization problems. Nowadays, BA has widely expanded its implementation in almost every area of optimization and engineering applications. This paper provides an updating literature review on applications and modifications of BA

\section{References}

[1] Almufti, S. (2017). Using Swarm Intelligence for solving NPHard Problems. Academic Journal of Nawroz University, 6(3), pp. 46-50. https://doi.org/10.25007/ainu.v6n3a78.

[2] Almufti, S., Marqas, R., \&Ashqi V., (2019). Taxonomy of bio-inspired optimization algorithms. Journal Of Advanced Computer Science \& Technology, 8(2), 23. https://doi.org/10.14419/jacst.v8i2.29402.

[3] Almufti, S. (2015). U-Turning Ant Colony Algorithm powered by Great Deluge Algorithm for the solution of TSP Problem. [online] Hdl.handle.net. Available at: http://hdl.handle.net/11129/1734 [Accessed 5 Aug. 2018].

[4] Agarwal, P., \& Mehta, S. (2014). Nature-Inspired Algorithms: State-of-Art, Problems and Prospects. International Journal of Computer Applications, 100(14), 14-21. https://doi.org/10.5120/17593-8331.

[5] Li, Y.: (2010), Solving TSP by an ACO- and -BOA-based Hybrid Algorithm. In: 2010 International Conference on Computer Application and System Modeling, pp. 189-192. IEEE Press,New York

[6] Yang, X.-S. (2010), A new metaheuristic bat-inspired algorithm. In Natureinspired cooperative strategies for optimization (pp. 65\{74). Springer. https://doi.org/10.1007/978-3-642-12538-6_6.

[7] Almufti S., \& Shaban A., (2018), U-Turning Ant Colony Algorithm for Solving Symmetric Traveling Salesman Problem, Academic Journal of Nawroz University, vol. 7, no. 4, pp. 45-49, Available: 10.25007/ajnu. v6n4a270. https://doi.org/10.25007/ajnu.v6n4a270.

[8] Almufti, S., R. Asaad, R., \& B. Salim, (2019). Review on Elephant Herding Optimization Algorithm Performance in Solving Optimization Problems. International Journal of Engineering \& Technology, 7(4), 6109-6114.

[9] Almufti, S., Marqas, R., \& Asaad, R. (2019). Comparative study between elephant herding optimization (EHO) and U-turning ant colony optimization (U-TACO) in solving symmetric traveling salesman problem (STSP). Journal Of Advanced Computer Science \& Technology, 8(2), 32. https://doi.org/10.14419/jacst.v8i2.29403.

[10] Asaad, R., Abdulnabi, N. (2018). Using Local Searches Algorithms with Ant Colony Optimization for the Solution of TSP Problems. Academic Journal ofNawroz University, 7(3), 1-6. https://doi.org/10.25007/ajnu.v7n3a193.

[11] Shi YH, Eberhart RC, (1998), A modified particle swarm optimizer[A],IEEE IntConf on Evalutionary Computation [C], pp. 63-73

[12] Almufti, S. (2019). Historical survey on metaheuristics algorithms. International Journal Of Scientific World, 7(1), 1. https://doi.org/10.14419/ijsw.v7i1.29497.

[13] Cui, Z., Sun, B., Wang, G., Xue, Y., Chen, J. (2017) A novel oriented cuckoo search algorithm to improve DV-Hop performance for cyberphysical systems. J. Parallel Distrib. Comput, 103, 42-52.https://doi.org/10.1016/j.jpdc.2016.10.011.

[14] Yang, X.S., Gandomi, A.H. (2012) Bat Algorithm: A Novel Approach for Global Engineering Optimization. Eng. Comput. 2012, 29, 464-483. https://doi.org/10.1108/02644401211235834.

[15] Bora, T.C., Coelho, L.D.S., Lebensztajn, L. (2012) Bat-Inspired Optimization Approach for the Brushless DC Wheel Motor Problem. IEEE Trans. Magn., 48, 947-950.https://doi.org/10.1109/TMAG.2011.2176108.

[16] Sambariya, D.K., Prasad, R. (2014) Robust tuning of power system stabilizer for small signal stability enhancement using metaheuristic bat algorithm. Int. J. Electr. Power Energy Syst., 61, 229-238.https://doi.org/10.1016/j.ijepes.2014.03.050.

[17] Sathya, M.R., Ansari, M.M.T. (2015) Load frequency control using Bat inspired algorithm based dual mode gain scheduling of PI controllers for interconnected power system. Int. J. Electr. Power Energy Syst., 64, 365-374.https://doi.org/10.1016/j.ijepes.2014.07.042.

[18] Sun, S., Xu, B. (2015) Node localization of wireless sensor networks based on hybrid bat-quasi-Newton algorithm. J. Comput. Appl., 11, 3842.https://doi.org/10.3991/ijoe.v11i6.5110.

[19] Cao, Y., Cui, Z., Li, F., Dai, C., Chen, W. (2014) Improved Low Energy Adaptive Clustering Hierarchy Protocol Based on Local Centroid Bat Algorithm. Sens. Lett., 12, 1372-1377.https://doi.org/10.1166/s1.2014.3355.

[20] Cui, Z., Cao, Y., Cai, X., Cai, J., Chen, J. (2017) Optimal LEACH protocol with modified bat algorithm for big data sensing systems in Internet of Things. J. Parallel Distrib. Comput.

[21] Cui, Z., Xue, F., Cai, X., Cao, Y., Wang, G.G., Chen, J. (2018) Detectin of malicious code variants based on deep learning. IEEE Trans. Ind. Inform., 14, 3187-3196.https://doi.org/10.1109/TII.2018.2822680.

[22] Hamidzadeh, J., Sadeghi, R., Namaei, N. (2017) Weighted Support Vector Data Description based on Chaotic Bat Algorithm. Appl. Soft Comput., 60, 540-551.https://doi.org/10.1016/j.asoc.2017.07.038.

[23] Alsalibi, B., Venkat, I.,Al-Betar,M.A. (2017)Amembrane-inspiredbatalgorithmtorecognizefacesinunconstrained scenarios. Eng. Appl. Artif. Intell., 64, 242-260.https://doi.org/10.1016/j.engappai.2017.06.018. 
[24] Cui, Z., Zhang, J., Wang, Y., Cao, Y., Cai, X., Zhang, W., Chen, J. (2019) A pigeon-inspired optimization algorithm for many-objective optimization problems. Sci. China Inf. Sci. https://doi.org/10.1007/s11432-018-9729-5.

[25] Tharwat, A., Hassanien, A.E., Elnaghi, B.E. (2016), A BA-based algorithm for parameter optimization of Support Vector Machine. Pattern Recognit. Lett., 93, 13-22.https://doi.org/10.1016/j.patrec.2016.10.007.

[26] Kashi S., Minuchehr A., Poursalehi N., \& Zolfaghari A., (2014). Bat algorithm for the fuel arrangement optimization of reactor core. Annals of Nuclear Energy, 64:144-151.https://doi.org/10.1016/j.anucene.2013.09.044.

[27] Alihodzic A. \& Tuba M., (2014). Improved bat algorithm applied to multilevel image thresholding. The Scientific World Journal, 2014, 2014.https://doi.org/10.1155/2014/176718.

[28] A. Latif and P. Palensky. Economic dispatch using modified bat algorithm. Algorithms, 7(3):328-338.https://doi.org/10.3390/a7030328.

[29] Taha A. M., Mustapha A., \& Chen S.-D., (2013). Naive bayes-guided bat algorithm for feature selection. The Scientific World Journal.https://doi.org/10.1155/2013/325973.

[30] Fister I., Rauter S., Yang X.-S., \& Ljubic K., (2014). Planning the sports training sessions with the bat algorithm. Neurocomputing.https://doi.org/10.1016/j.neucom.2014.07.034.

[31] Li Y. G. \& Peng J. P. (2014). An improved bat algorithm and its application in multiple ucavs. Applied Mechanics and Materials, 442:282286.https://doi.org/10.4028/www.scientific.net/AMM.442.282.

[32] Sambariya D. \& Prasad R., (2014). Robust tuning of power system stabilizer for small signal stability enhancement using metaheuristic bat algorithm. International Journal of Electrical Power \& Energy Systems, 61:229- 238.https://doi.org/10.1016/j.ijepes.2014.03.050.

[33] Cai X., Wang L.,Kang Q., \& Wu Q.,(2014). Bat algorithm with gaussian walk. International Journal of Bio-Inspired Computation, 6(3):166174.https://doi.org/10.1504/IJBIC.2014.062637.

[34] Kaveh A. \& Zakian P., (2014), Enhanced bat algorithm for optimal design of skeletal structures. Asian J Civial Eng, 15(2):179-212.

[35] Alsalibi, B., Venkat, I., \& Al-Betar, M. (2017). A membrane-inspired bat algorithm to recognize faces in unconstrained scenarios. Engineering Applications of Artificial Intelligence, 64, 242-260. https://doi.org/10.1016/j.engappai.2017.06.018.

[36] Nikov K., Nikov A. \& Sahai A., (2011), “A Fuzzy Bat Clustering Method for Ergonomic Screening of Office Workplaces”, Proceedings of Third International Conference on Software, Services and Semantic Technologies S3T, pp. 59-66.https://doi.org/10.1007/978-3-642-23163-6_9.

[37] Nakamura R., Pereira L., Costa K., Rodrigues D., Papa J. \& Yang X., (2012), "BBA: A Binary Bat Algorithm for Feature Selection”, Proceedings of XXV SIBGRAPI Conference on Graphics, Patterns and Images, pp. 291-297.https://doi.org/10.1109/SIBGRAPI.2012.47.

[38] Sabba S. \& Chikhi S., (2014), “A discrete binary version of bat algorithm for multidimensional knapsack problem", Int. J. BioInspired Computation, vol. 6, Issue 2, pp. 140-152. https://doi.org/10.1504/IJBIC.2014.060598.

[39] Zhang J. \& Wang G., (2012), "Image Matching Using a Bat Algorithm with Mutation", Applied Mechanics and Materials, vol. 203, Issue 2012, pp. 65-74.https://doi.org/10.4028/www.scientific.net/AMM.203.88.

[40] Fister I., Fister D. \& Yang X., (2013), "A hybrid bat algorithm”, Elektrotehniski vestnik.

[41] Xie J., Zhou Y. \& Chen H., (2013), “A Novel Bat Algorithm Based on Differential Operator and Lévy Flights Trajectory”, Computational Intelligence and Neuroscience, pp. 1-13. https://doi.org/10.1155/2013/453812.

[42] Afrabandpey H., Ghaffari M., Mirzaei A. \& Safayani M., (2014), "A novel bat algorithm based on chaos for optimization tasks", Proceedings of Intelligent Systems (ICIS), Iranian Conference, pp. 1-6.https://doi.org/10.1109/IranianCIS.2014.6802527.

[43] Gandomi A. \& Yang X., (2014), “Chaotic bat algorithm”, Journal of Computational Science, vol. 5, Issue 2, pp. 224232.https://doi.org/10.1016/j.jocs.2013.10.002.

[44] Yilmaz S., Kucuksille E. \& Cengiz Y., (2014), "Modified Bat Algorithm", Elektronika IR Elektrotechnika, vol. 20, Issue 2, pp. 7178.https://doi.org/10.5755/j01.eee.20.2.4762.

[45] Li L. \& Zhou Y., (2014), “A novel complex-valued bat algorithm”, Neural Computing and Applications, vol. 25, Issue 6, pp. 13691381.https://doi.org/10.1007/s00521-014-1624-y.

[46] Cai X., Wang L., Kang Q. \& Wu Q., (2014), "Bat algorithm with Gaussian walk", International Journal of Bio-Inspired Computation, vol. 6, Issue 3, pp. 166-174.https://doi.org/10.1504/IJBIC.2014.062637.

[47] Zhou Y., Xie J., Li L., \& Ma M., (2014), “Cloud Model Bat Algorithm”, The Scientific World Journal, pp. 111.https://doi.org/10.1155/2014/237102.

[48] Li D., Liu C. \& Gan W., (2011), "Proof of the heavy-tailed property of normal cloud model", Engineer and Science of China, vol. 13, Issue 4, pp. $20-23$.

[49] Dao T., Pan J., Nguyen T., Chu S. \& Shieh C., (2014), “Compact Bat Algorithm”, In: Intelligent Data analysis and its Applications. Volume II, Springer International Publishing: Cham, pp. 57-68.https://doi.org/10.1007/978-3-319-07773-4 6.

[50] Fister I., Fong S., Brest J. \& Fister I., (2014), “Towards the SelfAdaption of the Bat Algorithm”, Proceddings of the IASTED International Conference Artificial Intelligence and Applications (AIA 2014), pp. 400-406.

[51] Fister I., Fong S., Brest J. \& Fister I. (2014), “A Novel Hybrid SelfAdaptive Bat Algorithm”, The Scientific World Journal, pp. $112,2014$. https://doi.org/10.1155/2014/709738.

[52] Yilmaz S. \& Küçüksille E., (2015), “A new modification approach on bat algorithm for solving optimization problems”, Applied Soft Computing, vol. 28, pp. 259-275.https://doi.org/10.1016/j.asoc.2014.11.029.

[53] Jun L, Liheng L, \& Xianyi W., (2015), “A double-subpopulation variant of the bat algorithm. Applied Mathematics and Computation”. 263:361377. https://doi.org/10.1016/j.amc.2015.04.034.

[54] Meng X., Gao X., Liu Y. \& Zhang H., (2015), "A novel bat algorithm with habitat selection and Doppler effect in echoes for optimization", Expert Systems with Applications, vol. 42, Issue 17-18, pp. 6350-6364. https://doi.org/10.1016/j.eswa.2015.04.026.

[55] Wang G., Chu H. \& Mirjalili S., (2016), "Three-dimensional path planning for UCAV using an improved bat algorithm", Aerospace Science and Technology, vol. 49, pp. 231-238.https://doi.org/10.1016/j.ast.2015.11.040.

[56] Zhou Y., Luo Q., Xie J. \& Zheng H., (2016), “A Hybrid Bat Algorithm with Path Relinking for the Capacitated Vehicle Routing Problem”, In: Metaheuristics and Optimization in Civil Engineering, Vol. 7, pp. 255-276.https://doi.org/10.1007/978-3-319-26245-1_12.

[57] Cai X., Gao X. \& Xue Y., (2016), "Improved bat algorithm with optimal forage strategy and random disturbance strategy", International Journal of Bio-Inspired Computation, vol. 8, Issue 4, pp. 205214. https://doi.org/10.1504/IJBIC.2016.078666.

[58] Zhu B., Zhu W., Liu Z., Duan Q., \& Cao L., (2016), “A Novel QuantumBehaved Bat Algorithm with Mean Best Position Directed for Numerical Optimization", Computational Intelligence and Neuroscience, pp. 1-17. https://doi.org/10.1155/2016/6097484.

[59] Yammani C., Maheswarapu S., \& Matam S., (2016), "A Multi-objective Shuffled Bat algorithm for optimal placement and sizing of multi distributed generations with different load models", International Journal of Electrical Power \& Energy Systems, vol. 79, pp. 120131.https://doi.org/10.1109/TENCON.2016.7848354. 